\title{
Littérature et formation du lecteur : la dynamique de l'image dans la construction du sujet
}

\section{Françoise Demougin}

\section{OpenEdition \\ Journals}

Édition électronique

URL : http://journals.openedition.org/trema/664

DOI : 10.4000/trema.664

ISSN : 2107-0997

Éditeur

Faculté d'Éducation de l'université de Montpellier

Édition imprimée

Date de publication : 1 octobre 2005

Pagination : 27-35

ISSN : 1167-315X

Référence électronique

Françoise Demougin, « Littérature et formation du lecteur : la dynamique de l'image dans la construction du sujet », Tréma [En ligne], 24 | 2005, mis en ligne le 04 mars 2010, consulté le 30 avril 2019. URL : http://journals.openedition.org/trema/664; DOI : 10.4000/trema.664

Ce document a été généré automatiquement le 30 avril 2019

Trema 
Littérature et formation du lecteur : la dynamique de l'image dans la construction du sujet

Françoise Demougin

I. Le champ interrogé 
1 L"enseignement de la littérature est un objet de recherche qui convoque, comme toute recherche didactique et épistémologique, une recherche sur l'objet lui - même et sur les pratiques qui se sont greffées dessus, dans une perspective d'enseignement, de construction de savoirs ou de transmission de connaissances. Pourtant la littérature présente la particularité d'un objet non homogène, ne se définissant pas par un ensemble de savoirs cohérents rassemblés dans une construction théorique qui en garantit la validité. Il y a bien sûr l'histoire littéraire porteuse de savoirs spécifiques, mais aussi la critique, construite à partir de divers horizons, porteuses d'autres savoirs, et surtout les textes eux - mêmes, qui ne se constituent pas en objets de savoirs, ou alors de manière très problématique.

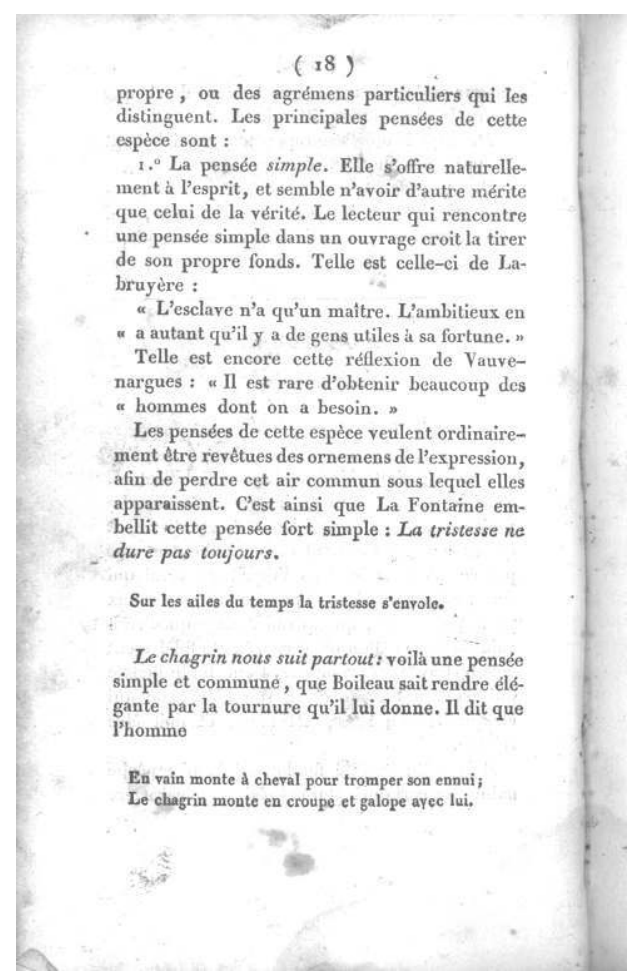
Qu'est - ce que savoir de la littérature ? En écrire, en réciter, connaître des dates et des auteurs, le contenu des ouvrages, analyser ces derniers, en parler, écrire des essais sur ces mêmes ouvrages ... Si la question ne peut être évacuée, elle ne peut pas non plus être véritablement traitée, mais juste décalée vers la question de sa didactique : la didactique de la littérature est spécifique à un domaine qui ne se constitue pas comme un champ de savoirs spécifiques, elle ne fournit aucun corps de doctrine ou aucune théorie de référence qui la légitime. Elle est, d'une certaine manière, auto - légitimée, par la proximité de son contenu à la pratique d'enseignement : BARTHES à CERISY disait que "la littérature c'est ce qui s'enseigne, un point c'est tout ». Ce vide théorique en son centre conduit la littérature à se référer à des modèles externes à sa pratique, et tout particulièrement sa didactique. La rhétorique a joué ce rôle fort longtemps, puis la linguistique a fourni les outils de la critique littéraire et le support aux démarches didactiques en littérature. Aujourd'hui la question est posée des limites de ce tout linguistique en littérature. L'anthropologie, non comme discipline constituée, mais comme champ de questionnement ne devient - elle pas alors le substrat de tout enseignement de la littérature?

\section{Les aspects méthodologiques}

2 A la différence des autres médiations culturelles, la littérature s'est construite pour l'essentiel dans l'Institution scolaire. Par ailleurs, elle reste disponible pour chaque sujet en tant que pratique individuelle de production et pas seulement en tant que pratique de réception. Autrement dit, la littérature est ce qui associe au plus près le sujet à sa langue et dans le même temps ce qui impose dans la langue une part considérable de codifications culturelles sélectionnées par l'Institution scolaire. A ce titre, les rapports entre littérature et langue, que cette dernière soit maternelle, étrangère ou seconde, ne peuvent être limités à une méthode ou à une situation d'apprentissage particulière. 
3 Lire, dire, écrire fait accéder à un double statut : celui, très culturalisé et collectif, que proposent les exercices sur la littérature et à propos d'elle; celui, beaucoup plus subjectif et personnel, de l'appropriation du discours de l'autre. Il nous faut reconnaître cette double postulation et en tirer les conséquences didactiques. Nous en dégagerons ici deux :

- d'une part, il est légitime de travailler sur les textes reconnus par l'Institution scolaire, dans les formes qu'elle leur accorde, du manuel au petit classique, et de viser par exemple deux types de productions écrites : celui du commentaire scolaire ou universitaire pratiqué dans la tradition des études littéraires françaises, celui aussi de la reproduction des formes littéraires classées selon genres et types de textes. L'objectif n'est pas tant alors de maîtriser les savoir - faire liés à ces formes écrites mais de s'approprier quelques éléments d'une culture institutionnelle.

- d'autre part, il est tout aussi légitime de laisser l'élève construire dans la langue une part de son expérience. Les écrits visés, pour rester dans l'écrit, n'ont pas lieu de répondre à des critères savants. Il peut s'agir de simples listes avec les premiers mots appris comme de journaux intimes ou de petits récits dans lesquels s'expérimente, dans la langue de l'autre, la construction du sujet. La démarche est par essence littéraire. Il ne s'agit plus de se frotter à une culture littéraire française mais de se construire, dans la langue, une nouvelle médiation au monde.

4 La question à se poser n'est donc pas tant celle du niveau des élèves, surtout s'il s'agit d'élèves en difficulté, niveau toujours susceptible de justifier une mise à l'écart de la littérature, mais bien celle du rapport construit à la littérature dans la langue maternelle avant d'en évaluer les conséquences en terme de choix d'activités, de démarches et de corpus. Il s'agit là de construire, ou plutôt de prendre en compte la construction d'un rapport intime à la lecture et à l'écriture littéraires dont on conçoit bien qu'il est à la limite du champ didactique et qu'il implique des relations intersubjectives complexes, passant par l'évitement de l'obstacle du «je n'ai pas assez de connaissances pour ...». Le texte littéraire, en effet, est un lieu stratégique essentiel, à la jonction du cognitif et du culturel, mettant en jeu des compétences à la fois langagières et culturelles. Dans cette perspective, il est porteur de symboles et de valeurs, point nodal de la communication entre les individus d'une même société et cette fonction sociale, souvent méconnue, n'est pas la moindre. Mais aussi, et peut - être surtout, il est un lieu où le sujet se construit dans sa capacité à s'approprier une expérience littéraire du texte, à la reconstruire et la prolonger. L'hypothèse anthropologique se trouve ici confirmée. Le texte littéraire ne sert pas à évaluer une compétence de lecture, il apprend à penser, à se construire comme sujet lecteur. Dans cette perspective, il importe que la lecture ne constitue pas un obstacle matériel pour l'apprenant.

5 L'enseignement de la littérature peut se concevoir dans divers cadres : en français langue maternelle, dans le cadre d'une discipline scolaire qui s'est imposée relativement récemment, le Français - Lettres, qui résume dans son hésitation toute l'ambiguïté de la situation dans le second degré (on enseigne la langue et la littérature), mais aussi dans le cadre de l'enseignement du français langue étrangère, ou dans le cadre plus récent de l'école primaire en France ${ }^{1}$. Ces cadres institutionnels délimitent les champs d'observation sur lesquels nous travaillons.

6 Vient ensuite la question des observables et des outils d'évaluation. La reconfiguration du champ des études littéraires atteste aujourd'hui de l'importance que nous soulignons ici de l'anthropologique, qui se profile dans ces trois dimensions, de manière encore conflictuelle : 
7 les pratiques prescrites et les outils constituent un premier faisceau de données: programmes, manuels, revues et ouvrages didactiques pour l'essentiel, qui doivent être analysés comme des constructions discursives, comme des objets porteurs de représentations, de théorisations implicites ou explicites. Ils montrent bien des tensions entre les logiques formalistes centrées sur des objets linguistiques et des logiques anthropologiques centrées sur le sujet.

8 les pratiques réelles constituent un deuxième domaine de travail : celles de l'enseignant, des élèves, analysables à travers des situations, des interactions et des productions langagières (orales et écrites), des représentations. A travers ces différents axes, on peut repérer plus précisément les domaines de référence convoqués dans les pratiques prescrites ou réelles: champs théoriques, conceptualisations diverses qui orientent l'activité et lui donnent sens. Les champs de référence sont en nette évolution : tous les travaux sur la lecture, sur la démarche interprétative ou sur la notion de texte, à laquelle on pourrait substituer la question de la parole, montrent l'émergence d'un nouveau domaine de référence, en prise avec des questions plus anthropologiques que linguistiques.

9 les pratiques langagières et sociales qui interfèrent, notamment la lecture et l'écriture, qui dépassent le champ scolaire et le seul enseignement de la littérature, de ses usages sociaux et de ses implications psycho - affectives. Ces pratiques et ces représentations mises en jeu soulignent les facteurs humains et langagiers dans ces expériences.

\section{La dynamique de l'image}

\section{III.1. Littérature de jeunesse et image}

Cette perspective globale, dans laquelle s'inscrit notre réflexion, trouve en la littérature de jeunesse, qui appartient, sans conteste aujourd'hui, au champ de la littérature, et dans l'album en particulier, un terrain d'investigation précieux. La littérature de jeunesse ${ }^{2}$ présente en effet une langue facile d'accès, sans interprétation pré - construite, offrant une aire de négociation du sens particulièrement riche ${ }^{3}$. La présence d'une trame narrative structurante y constitue un lien précieux avec l'expérience personnelle de l'élève ${ }^{4}$ en même temps qu'elle aide à la compréhension et la mémorisation, donc à l'échange. Enfin, et surtout peut - être, la présence d'images, au sein du rapport texte / image, permet de profiter des apports de l'image, sur le plan de l'implicitation notamment. L'image, en effet, dans son rapport au texte, permet à l'élève de passer outre des difficultés langagières, ou psychosociales, et d'avoir accès à une compréhension fine des valeurs exprimées par l'œuvre. Elle va aider à combler des lacunes (linguistiques surtout), à complexifier la lecture (en habituant l'œil à un parcours heuristique), à susciter le débat (elle a valeur d'échange, comme la définissait le réalisateur R. BRESSON en 1957), à permettre l'itération fondamentale: réalités, mots, images dans la constitution d'images mentales nécessaires à tout apprentissage.

\section{III.2. Sujet lecteur et image}

11 Si la question actuelle est bien de former un sujet lecteur, dans l'apprentissage de la langue, l'examen des conditions de cette (trans) formation au cours de laquelle le sujet doit se déplacer, faire varier un point de vue et se construire dans ce mouvement, montre 
l'image comme un outil essentiel pour accompagner la mise en activité du sujet lecteur. L'état de vigilance provoqué par la présence dans l'image d'éléments signifiants, que le texte ne livre pas d'emblée, permet à l'élève, avant les mots, d'entrer dans la transformation du contingent en signification. Que se passe - $t$ - il au cours des échanges entre élèves développés lors de lectures d'albums? La recherche collective de sens se fait au sein d'une sorte d'euphorie langagière dans laquelle la vivacité n'empêche pas la cohérence, dans laquelle chacun rebondit sur la parole de l'autre. Le gain est autant sémantique, cognitif et rationnel que relationnel. Chacun bénéficie du travail collectif en intégrant le point de vue de l'autre. Expériences esthétique et cognitive ${ }^{5}$ vont de pair. Le moi se décentre, s'élargit vers un soi réflexif plus ouvert aux représentations de l'autre. Le développement de ces capacités langagières et réflexives ${ }^{6}$ n'est certes pas dû à la seule présence des images mais le lecteur prend facilement, face à l'image, une posture d'enquêteur à la recherche d'indices, qui génère une méfiance, un doute face à une pseudo - vérité de la représentation, et par là une activité négociatrice. La relecture de l'album, par exemple, est souvent spontanément demandée et acceptée par la communauté classe comme une activité sensée ${ }^{7}$ et validante.

12 La lecture de l'image met en place une socialisation effective: l'analyse progresse, par stratification, grâce à l'apport de chacun. Les conflits cognitifs finissent par être surmontés à partir d'une capacité à intégrer, au - delà des antagonismes, les propositions des interlocuteurs présents. Certes il faut en amont un travail d'institution du cadre du débat argumentatif et démocratique qui permette la socialisation cognitive des élèves. Cependant la nature polysémique voire énigmatique ou encore dérangeante de l'image interrogée, qui ne rend pourtant pas l'élève mutique parce qu'elle va de pair paradoxalement avec une dimension proximisante qui le rassure, semble aider à une prise de conscience de la nécessité de l'autre dans la quête du sens et à la construction d'un espace interlocutif. L'ordonnancement et la pacification de ce dernier restent sans doute à construire mais peuvent s'enraciner précisément dans les désaccords induits par la pluralité des interprétations possibles: chaque élève est appelé à créer des significations, des cognitions, des rapports de place, des identités. Sur un plan subjectif, chacun, institué comme interlocuteur valable, peut trouver à s'identifier dans sa ressemblance (idem) et sa différence (ipse) avec les autres, en persévérant dans l' » être ensemble " sans se laisser enfermé dans la sérialité. Des outils tels que le carnet de lecture, des activités telles que la table ronde, le débat, des cadres tels que l'organisation d'un prix littéraire interclasses, sont précieux dans cette perspective. Nul n'est alors habilité prioritairement à dialoguer avec le texte. Ainsi sont amenés à se développer à la fois l'intrasubjectif et l'intersubjectif du je en action dans la communication scolaire.

Les discussions que nous avons enregistrées lors de multiples expérimentations ${ }^{8}$ montrent que les élèves ne différencient pas, dans la construction du sens, les mots de l'image, plaçant en premier dans leur préoccupation le processus de création commun aux deux modes d'expression. L'image, parce que sa lecture a intégré le décodage d'éléments symboliques, habitue l'œil à une démarche heuristique, en conférant à l'élève un regard et non plus seulement une perception. On passe à la prise en compte progressive d'une signification secondaire, interprétative, de l'image. Une capacité réelle à prendre des risques interprétatifs ( ça veut dire ...»), à manifester le souci d'en vérifier la validité, se développe, dans la prise de conscience d'un décalage entre ce qui est vu, décrit, compris et ce qui peut être signifié. 

posé avec son propre monde, par un processus d'actualisation des données textuelles, prépare le jeune lecteur à l'idée d'une non gratuité de la lecture dont la dimension strictement scolaire s'estompe. L'incomplétude du monde fictionnel se trouve en quelque sorte compensée par le caractère analogique de l'image qui force le lecteur à restaurer un mode mimétique de la perception. Et si l'élève perçoit bien une mise en signification volontaire de l'image, comme du texte, par l'auteur, il la dépasse aussi. Placé dans une position de secondarité critique face à l'album, parce que l'image l'a obligé à juxtaposer deux modes de lecture : le lire et le voir, il pressent que ce qui se joue dans l'image et dans le texte, dans le langage par conséquent, excède aussi ce qu'y peut mettre l'auteur et que c'est alors au lecteur d'exister, par et dans sa propre parole. lecteur comme une seule activité distanciée. Est - il encore besoin de souligner la nécessité, ailleurs parfaitement démontrée ${ }^{9}$, de conjuguer lecture distanciée et lecture émotionnelle? Or, précisément, le rapport texte / image induit cette double lecture. Un processus d'empathie se trouve par nature enclenché dans et par l'image. L'axiologie du lecteur est sollicitée par le visuel, par la dimension proximisante de l'image. Tout se passe comme si l'image permettait au personnage de l'album de s'ouvrir plus complètement au lecteur : simplement écrit, le personnage resterait une abstraction tandis que, montré, il devient aussi une réalité appréhendée dans sa singularité et son évidence. De ce point de vue, l'image empêche la lecture des élèves de se restreindre à une suite d'inférences : elle donne, ou redonne, une place à l'intuition dans le parcours interprétatif. Et ce d'autant plus que la nature spatiale de l'image facilite l'exercice subjectif de la mise en relation. L'élève apprend par l'image à "correspondre » avec le texte, à exprimer par tout son réseau perceptif ce qu'il comprend, ressent, et anticipe. Le texte que l'élève a devant lui présent, c'est l'image qui lui permet de le lier à une expérience passée et à une projection narrative et ontologique.

\section{III.3. Lire un texte littéraire}

Dans le même temps, par conséquent, l'image conjugue simultanément le réel et le non rée $^{10}$ en ce qu'elle est à la fois analogie, ressemblance et construction pure. C'est bien alors le statut de la vérité du texte littéraire qui est en jeu. L'élève ne peut plus se réfugier derrière l'abstraction des mots, ou le caractère fictif des personnages puisque l'image, par son autorité, donne un sens à la fiction. Dans le double rapport au réel qui vient d'être évoqué (être et ne pas être), l'image, remet en place le lecteur dans son rapport à la fiction. Elle empêche un processus d'identification totale, mécanique, tout comme elle empêche aussi une extériorité radicale : ce faisant elle le libère d'une double aliénation. Dans le même temps, elle permet l'évitement du textualisme comme celui du subjectivisme.

17 L'image parvient ainsi à questionner le statut du lecteur, à travailler l'épaisseur intertextuelle, à faire penser le lecteur en réseau. Il s'agit là d'une opération intellectuelle fondamentale, qui permet aux élèves de comparer, d'associer, de dissocier, d'expliciter et de déduire, si cela est possible, des règles qui seront par la suite stabilisées et formalisées. Elle développe des conduites à la fois individuelles et interactives, et elle est la part de plus en plus active prise par l'élève en écho aux lectures qui lui sont proposées. Ce dernier fait ainsi partager son cheminement, sa propre démarche de "pensée en réseau ». Ces

Tréma, 24 | 2009 
apprentissages s'inscrivent dans la durée et excluent toute exploitation didactique hâtive. Le sujet culturel y est sans cesse sollicité, invité à rencontrer des objets de savoirs complexes nécessaires à la construction de ses apprentissages, à la compréhension du processus de production de sens. Ce faisant, le lecteur est amené à jouer sans réticence sur le réel et ce jeu informe son retour au texte: la complexité des mots ne tient plus seulement à un déchiffrement mais à la certitude qu'ils peuvent dire plus qu'on ne lit.

La lecture devient une expérience complexe où sensation et réflexion se trouvent mêlées, et dans le même temps dépassées toutes les deux. Nécessaire à la pensée ${ }^{11}$, l'image n'est vraiment ni sensation, ni réflexion : elle suppose la première puisqu'elle procède à partir d'elle, et elle est nécessaire à la seconde. L'image, entre le réel et l'intellect, apparaît donc comme un principe dynamique qui ouvre l'accès à la réalité qu'elle produit, c'est - à - dire la réalité du sujet lisant qui se construit dans un aller - retour du réflexif et de l'affectif. Montrer au jeune lecteur une image du personnage de l'histoire qu'il est en train de lire ne l'empêche pas de "penser » le personnage, contrairement au reproche trop souvent fait à la mise en image de restreindre la conscience interprétative : l'image offerte au lecteur atteste au contraire de la possibilité qui lui est faite de créer un monde nouveau. Donner à voir fait donc retourner le lecteur au texte de manière gourmande (l'élève reporte sur le texte le plaisir pris à la lecture de l'image, de ses codes, de ses implicites,) et "sans cesse renouvelée» (l'élève effectue un aller - retour constant entre monde fictionnel et expérience personnelle forcément changeante), qui ne réduit pas la lecture à la simple possibilité de revivre une situation passée : l'émotion de la lecture constitue bien chaque fois "non une réanimation d'émotions déjà vécues, mais une expérience neuve, irremplaçable », selon les mots de GRACQ ${ }^{12}$.

Entre le contexte pragmatique et le contexte sémantique, entre l'usage cognitif et pédagogique de l'image, tourné vers les choses et l'usage affectif et dynamique, tourné vers les hommes, il nous faut sans doute placer l'image dans la mise en relation du texte et du sujet lecteur demeurés jusque là étrangers l'un à l'autre, ou canalisés dans un rapport imposé, pré - construit scolairement. Toutefois c'est bien

l'activité du sujet qui devient première dans l'acte de lecture, pouvant alors se déployer en dehors même du texte lui - même ${ }^{13}$. Il s'agit bien pour le lecteur de reconnaître ses émotions devant les images et le texte, de se construire à travers le dedans et le devant de l'image, deux postulations indispensables au bonheur de la distance critique ${ }^{14}$.

L'activité du sujet lecteur ne consiste pas à « réanimer " des signes. Pas davantage à donner prise à l'illusion. Le retour réflexif, amené par l'image, du lecteur au texte, ce retour à un texte qu'il découvre autrement, cette réinscription du lire dans un vivre, cette prise de conscience de la singularité de sa rencontre avec l'œuvre, conduit au contraire à une véritable dynamisation de la lecture, une construction de soi dans la pédagogie de l'autre, dont la conséquence didactique semble bien être que parler du texte, de son fonctionnement, n'est pas l'accès obligé à ce dernier, semble bien être que prendre en compte le caractère intime de la communication avec un livre est une nécessité. Jusqu'où et comment cette intimité peut - elle avoir sa place, être provoquée, voire développée à l'école? La question est aujourd'hui posée ${ }^{15}$.

\section{Un enjeu essentiel}

L'enjeu ultime, et grave, d'une telle lecture participante de l'élève est de combattre la «misère symbolique $»^{16}$, qui nous guette, c'est - à - dire une perte d'individuation 
résultant d'une perte de participation, précisément, à la production et au maniement des symboles, ces derniers désignant autant les fruits de la vie intellective (concepts, savoirs) que ceux de la vie sensible (arts, savoir - faire). Par son fait, le lecteur est le rapport à ce qu'il lit en tant qu'il est « singulier », et non standardisé en " particulier » renvoyant à des communautarismes en tous genres. Car la particularisation n'est rien d'autre qu'une annulation du singulier ${ }^{17}$, qui aboutit à faire du lecteur spectateur le même que son voisin dans une appartenance à un groupe donné. Le lecteur non participant risque de tomber dans le piège de textes recommandés par l'Institution, ou par l'enseignant, traités en amont par eux ; il risque, répétant une signification construite en dehors de lui, répétée par plusieurs, de perdre cette singularité qui le constitue pensant et existant, de devenir un simple consommateur et par là d'échapper à la communauté constituée « originairement dans l'intimité du lien de soi à soi »"

La littérature, en tant qu'activité artistique, est précisément le lieu, l'expérience même de la singularité sensible comme invitation à l'activité symbolique. Le travail sur le texte littéraire, sur l'image qui l'accompagne, délivrant le désir du canal où la consommation l'a enfermé, libère en même temps les capacités mentales, intellectuelles, affectives et esthétiques du sujet. Savoir interpréter, autrement dit savoir discerner, juger (krinein), revient bien à rétablir le circuit du désir, par conséquent du don, et par là à éviter la décomposition du social à proprement parler. On le voit, la question didactique rejoint ici la question esthétique et la question politique, dans la question de la relation à l'autre au cœur d'un "sentir ensemble". Dans une même ambition: redonner à tous le sens de l'expérience esthétique, de manière à retrouver, en soi comme en nous, un devenir porteur d'avenir.

\section{BIBLIOGRAPHIE}

BORELLE M., GOULETTE J. P., (2002), Cognition et création. Liège : Mardaga.

BOUGNOUX D., (2002), Introduction aux sciences de la communication. Paris : La Découverte.

CHABANNE J. C., (2002), « Les évolutions récentes du français à l'école primaire ", Tréma n¹9

Littérature enseignée : reconfigurations, IUFM Montpellier, p. 37 - 54.

CORBEVOIS, DEVANNE, DUPUY, MARTEL, (2000), Apprentissage de la langue et conduites culturelles à

l'école maternelle. Paris : Bordas Pédagogie.

DELEUZE G., (1983), Cinéma I., L'Image - mouvement. Paris : Minuit.

GAMBIER A. (dir.), (2003), Les Dons de l'image. Paris : L'Harmattan.

GOODY J., (2003), La Peur des représentations, 1997, édition française. Paris : La Découverte.

GRACQ J., (1988), En lisant en écrivant. Paris : Corti.

JOUVE V., (1992), L'effet - personnage dans le roman. Paris : PUF.

LAUXEROIS J., (2002), « A titre amical », postface à L'Amicalité, A propos.

RICCEUR P., (1969), Le conflit des interprétations. Essais d'herméneutique. Paris : Seuil.

STIEGLER B., (2004), De la misère symbolique : 1 - L'époque hyper industrielle. Paris : Galilée.

TAUVERON C. (dir.), (2002), Lire la littérature à l'école. Paris : Hatier.

TISSERON S., (2002), Les bienfaits des images. Paris : O. Jacob. 


\section{NOTES}

1. Les programmes 2002 instaurent un enseignement de la littérature à l'école.

2. Il n'est pas dans notre propos de la définir mais plusieurs traits que nous allons mettre en avant constituent autant de caractérisations.

3. Précisément la «marge d'interprétation » plus vaste laissée au lecteur selon les termes de M. TOURNIER évoquant le caractère pour lui plus littéraire du texte dans Vendredi ou la vie sauvage que dans Vendredi ou les limbes du Pacifique.

4. « Mettre en récit, c'est donner sens à l'expérience humaine » dirait P. RICOEUR.

5. Comme l'ont mis en valeur les travaux d'Aristote déjà, et plus près de nous de N. GOODMAN.

6. Voir les travaux de G. H. MEAD à ce sujet.

7. Ces éléments ont été développés dans une communication que nous avons faite lors du colloque Sujets lecteurs et enseignement de la littérature qui s'est tenu à Rennes en janvier 2004 : « Lecture d'images et (re) positionnement du lecteur ».

8. Notamment dans le cadre du projet innovant « littérature et image » développé sur le site de Nîmes depuis septembre 2003 (pilote : F. DEMOUGIN).

9. Voir, par exemple les propos de C. TAUVERON dans Lire la littérature à l'école.

10. J. GOODY dans La Peur des représentations (Ed. française, Paris, La Découverte, 2003) évoque «l'ambivalence des images », celle qui naît de l'aptitude de ces dernières à tout à la fois être et ne pas être la chose ou l'idée ainsi rendue présente.

11. Voir ARISTOTE déjà, De anima, livre III.

12. Voir notamment En lisant en écrivant, Corti, 1988.

13. Voir Deleuze : entretien avec S. DANEY, journaliste à Libération, en 1983 à l'occasion de la parution de son livre Cinéma I L'Image - mouvement (Paris, Minuit, 1983) : «Une image ne vaut que par les pensées qu'elle crée. Dans les images que vous distinguez, l'image plane n'est pas séparable d'une pensée qui réagit sur elle, et qui varie d'ailleurs avec les auteurs (...) ». L'image ainsi apparaissait au philosophe comme un lieu de pensée c'est - à-dire permettant la "remontée formelle de la situation à une question enfouie" : des données d'une situation très urgente aux données d'une question, encore plus urgente, cadrée dans la situation.

14. Voir à ce sujet une source de réflexion possible pour les enseignants dans les travaux du psychanalyste S. TISSERON, directeur de recherche, Paris X - Nanterre et en particulier dans son dernier ouvrage : Les bienfaits des images, Paris, O. Jacob, 2002. S. TISSERON propose de travailler avec les jeunes spectateurs d'images sur le langage, les scénarios intérieurs et les gestes qui sont autant de mise en scène des effets corporels et émotionnels des images sur eux.

15. Certains éléments de réponse ont été apportés dans le primaire, par des modalités de travail, comme la mise en réseaux, les écrits et oraux réactifs, le souvenir de lecture différé, dont l'objectif commun est d'arrimer la culture apportée à l'expérience personnelle du sujet.

16. Voir l'ouvrage de B. STIEGLER : De la misère symbolique, Paris, Galilée, 2004.

17. Op. cit. p. 26.

18. Voir J. LAUXEROIS, « A titre amical », postface à L'Amicalité, A propos, 2002, p. 85. 


\section{RÉSUMÉS}

Le texte littéraire apparaît comme un lieu où le lecteur se construit comme sujet en apprenant à penser, à reconstruire et prolonger une expérience littéraire. La lecture de l'image, en particulier dans la littérature de jeunesse, est emblématique de cette construction du sujet. Chaque rencontre avec une œuvre est singulière, et cette prise de conscience conduit à une véritable dynamisation de la lecture, conçue dès lors comme "participante ", dont l'enjeu est de combattre le risque d'une misère symbolique, au cœur d'une ambition qui est de redonner à tous le sens de l'expérience esthétique, de manière à retrouver, en soi comme en nous, un devenir porteur d'avenir.

We consider that literary texts are places where readers build themselves as subjects, when they are taught how to think, (re)construct and extend meaning from literary experience. "Reading " a picture, particularly in youth literature, is emblematic of personal fulfilment. And, since each encounter with a work is unique, awareness raising should provide true dynamics in the reading process, thus to be thought of as involving and participative. Countering the risk of aesthetic paucity is a challenge, the ambitious aim of which is to lead everyone to gain a sense of aesthetic experience, within themselves as well as within ourselves, so as to let the road open to a more promising future.

INDEX

Mots-clés : anthropologie, image, littérature, sujet lecteur, symbole

Keywords : anthropology, literature, picture, reader as a subject, symbol

\section{AUTEUR}

\section{FRANÇOISE DEMOUGIN}

Maître de conférences en lettres, IUFM Montpellier 